Pract. oto-rhino-laryng. 1969;31:1-2

\title{
Preface Vorwort Avant-Propos
}

As from 1969 Practica ORL· will undergo several changes. The aim of these alterations is to enable the journal to play a more significant role in the sphere of international scientific cooperation.

With this in view, the Editorial Board will be enlarged to include new collaborators from various countries, thus providing a broader base.

Because English is becoming more and more the main international language in the field of medicine, in future an increasing number of English papers will be published. Eor the same reason, all the papers will include, in addition to a summary written in the original language of the paper, an English abstract, which will appear at the beginning of the article.

In future key words will be given at the top of each paper. These will indicate the contents of the paper at a glance and will increase its scientific value.

We hope that these alterations will make Practica ORL - even more valuable to its subscribers.

Vorwort

Vom Jahre 1969 an soil Practica ORL - mil dem Ziel umgestal-tet werden, daß die Zeitschrift in verstärktem Maße der inter-nationalen wissenschaftlichen Zusammenarbeit dienen kann.

$\mathrm{Zu}$ diesem Zwecke wird der Redaktionsstab durch neue Mit-arbeiter aus verschiedenen L-ändern erweitert, um ihn so auf eíne breitere Basis zu stellen.

Da sich das Englische immer mehr zur internationalen Sprache der Medizin entwickelt hat, sollen in Zukunft Arbeiten in engli-scher Sprache in vermehrtem Umfang veröffentlicht werden. Aus dem selben Grund erhalten sämtliche Arbeiten neben einer $\mathrm{Zu}$-sammenfassung in der Originalsprache ein englisches «Abstract», das an der Spitze der Veröffentlichung stehen wird. Am Kopfe einer jeden Arbeit erscheinen in Zukunft Key Words, die einen schnellen Überblick über den Inhalt der Arbeiten gewäh-ren und die wissenschaftliche Ausnützung erleichtern. Wir hoffen, daß diese Änderungen die Practica ORL· für den Abonnenten noch wertvoller machen werden.

Avant-Propos

La réforme projetée pour 1969 dans la presentation de Prac-tica ORL a pour but de lui conférer un rang notoire dans les milieux scientifiques internationaux.

Ann de renforcer sa position, de nouveaux membres, origi-naires de divers pays, ont été incorporés au sein du Comité de Redaction.

D'autre part, Гanglais étant de plus en plus reconnu comme langue Internationale dans le domaine de la médecine, un nombre croissant de travaux anglais seront publiés à $\Gamma$ avenir. Pour cette même raison, tous les travaux comprendront dorénavant, en plus du resume rédigé dans la langue originale. un « abstract» anglais place au debut de $\Gamma$ article.

De plus, des « Key Words » seront publiés en tête de chaque article ; ces derniers permettront d'orienter le lecteur en un clin d'æil sur le contenu du travail et d'accroître ainsi sa valeur scientifique.

Nous espérons que ces changements seront appréciés par les abonnés et qu'ils leur rendront de précieux services. 